Part of Journal of Research of the National Bureau of Standards, Volume 35, October 1945

\title{
COLOR STANDARD FOR RUBY MICA
}

\author{
By Deane B. Judd
}

\section{ABSTRACT}

At the request of the Metals Reserve Company and the War Production Board, a study has been made of the color classification of mica used by the mica-consuming industry. This classification has been found to be consistent and can be reproduced from the chromaticity of the mica specimen and its daylight transmission considered in relation to thickness. A method of defining ruby mica in these terms is described, and as it does not depend upon the physical integrity of any material color standard, this definition constitutes a fundamental color standard for ruby mica. This standard is particularly for application to micas close to the border line between ruby and nonruby.

\section{CONTENTS}

I. Introduction

II. Selection of borderline specimens

III. Method of measurement

IV. Derivation of a hue and a lightness index for mica colors

V. Derivation and validification of a color standard for ruby mica..... 251

VI. Procedure used in color grading mica _...

\section{INTRODUCTION}

Mica is a critical material for the manufacture of electrical equipment for the armed forces because it is a good insulator and can be split to desirable thicknesses. Capacitor manufacturers prefer "ruby" mica because they find that the better qualities (clear, clear and slightly stained, fair stained, good stained $)^{1}$ are of satisfactory power factor and easy to split, whereas the "nonruby" micas of the same qualities often tend to be brittle, and much of it is found to have undesirably high power factor. At the request of the War Production Board and the Metals Reserve Company, a study has been made of the system of determining the color of mica by visual inspection used by the mica-consuming industry. There are micas of many colors: light green, green, rum, ruby, and intermediates between them, so that classification into ruby and nonruby often depends upon color differences so small as to be scarcely perceptible. As there have been no formally accepted standards for ruby mica the inspector must develop from his experience an idea of the borderline colors, and remember them. Furthermore, a given borderline mica will exhibit a progression of colors from nearly colorless through yellowish gray to dark

\footnotetext{
${ }^{1}$ Standard methods of test for grading and classification of natural mica, Am. Soc. Testing Materials, Standards, part III, Nonmetallic Materials, p. 504 (1944) D 351-38.
} 
brown, inclining more toward red as the thickness of the specimen viewed is increased; so the inspector must take into account the thickness of the specimen in order to arrive at a judgment characteristic of the mica independent of its thickness. This he does by viewing the mica in several ways; single specimens of various thicknesses viewed against the sky, a handful of specimens viewed against the sky, single specimens of various thicknesses placed on a white surface and viewed by light that passes through each specimen twice, once to illuminate the white surface, once to reach the eye of the observer, and finally directly in the stock box or barrel in an opaque layer by light reflected from the nearly parallel faces of the various specimens in random position at various depths in the pile. His judgment is complicated by the fact that mica from a single mine to be classified exhibits more or less variation in color from specimen to specimen and from spot to spot within the same specimen.

In spite of these difficulties there has become established by trade practice a fairly definite idea of what is acceptable to the micaconsuming industry as ruby mica. An experienced inspector of mica comes to remember the family of colors corresponding to specimens of various thicknesses by constant intercomparison of members of such families. He disregards small variations such as air or mineral inclusions, or cloudy or stained areas of a specimen, makes a mental a verage of the colors of various specimens from a given lot or mine, and makes a mental comparison with a mental border line between ruby and nonruby that is surprisingly reliable and consistent.

It is the purpose of this paper to set up in fundamental terms the color standard for ruby mica, heretofore existent only in the memories of mica inspectors, and to show how this fundamental standard may be applied, particularly to specimens so close to the border line between ruby and nonruby as to make doubtful their classifications by direct visual inspection.

\section{SELECTION OF BORDERLINE SPECIMENS}

At the request of the War Production Board an industry task committee familiar with the visual classification of mica met in Asheville, N. C., on December 4, 1944, to review the classification of 35 lots of mica, classed as nonruby or doubtful, at the southern headquarters of the Colonial Mica Corporation, set up as agent for the Metals Reserve Company to ensure so far as possible a maximum supply of domestic mica for war industries. One-pound samples were taken of each of these lots and an extra sample of three of them. In addition, specimens characteristic of near-borderline mica from nine mines and eight stock boxes were also selected. These 17 specimens were visually intercompared, and it was noted that the color grading was consistent to a high degree. After correction of one small inconsistency in the color grading by consultation with George Purcell, Wm. J. Alexander, and Bradley Johnson of the Colonial Mica Corporation, with due regard to the judgments of the industry task committee, five specimens were selected to represent the border line between ruby and nonruby mica. These specimens were cut in two and each half marked respectively as follows: No. 2 quality border line, No. 1 quality border line, No. 1 quality ruby, No. 37 green, and No. 17 border line. One half of each was left at the Asheville offices as a 
physical standard defining ruby mica. The other half of each was measured to provide a fundamental definition of the border line between ruby and nonruby mica.

\section{METHOD OF MEASUREMENT}

The approximate thicknesses of the five standard specimens were found by means of a direct-reading micrometer.

The five specimens were illuminated perpendicularly by artificial daylight (incandescent lamp combined with Corning Daylite glass of such thickness as to yield a color temperature of $6,500^{\circ} \mathrm{K}$ ) in the chromaticity-difference colorimeter ${ }^{2}$ and the chromaticity difference between the incident and emergent light was determined for each specimen in terms of the chromaticity coordinates $(x, y)$ of the stand-

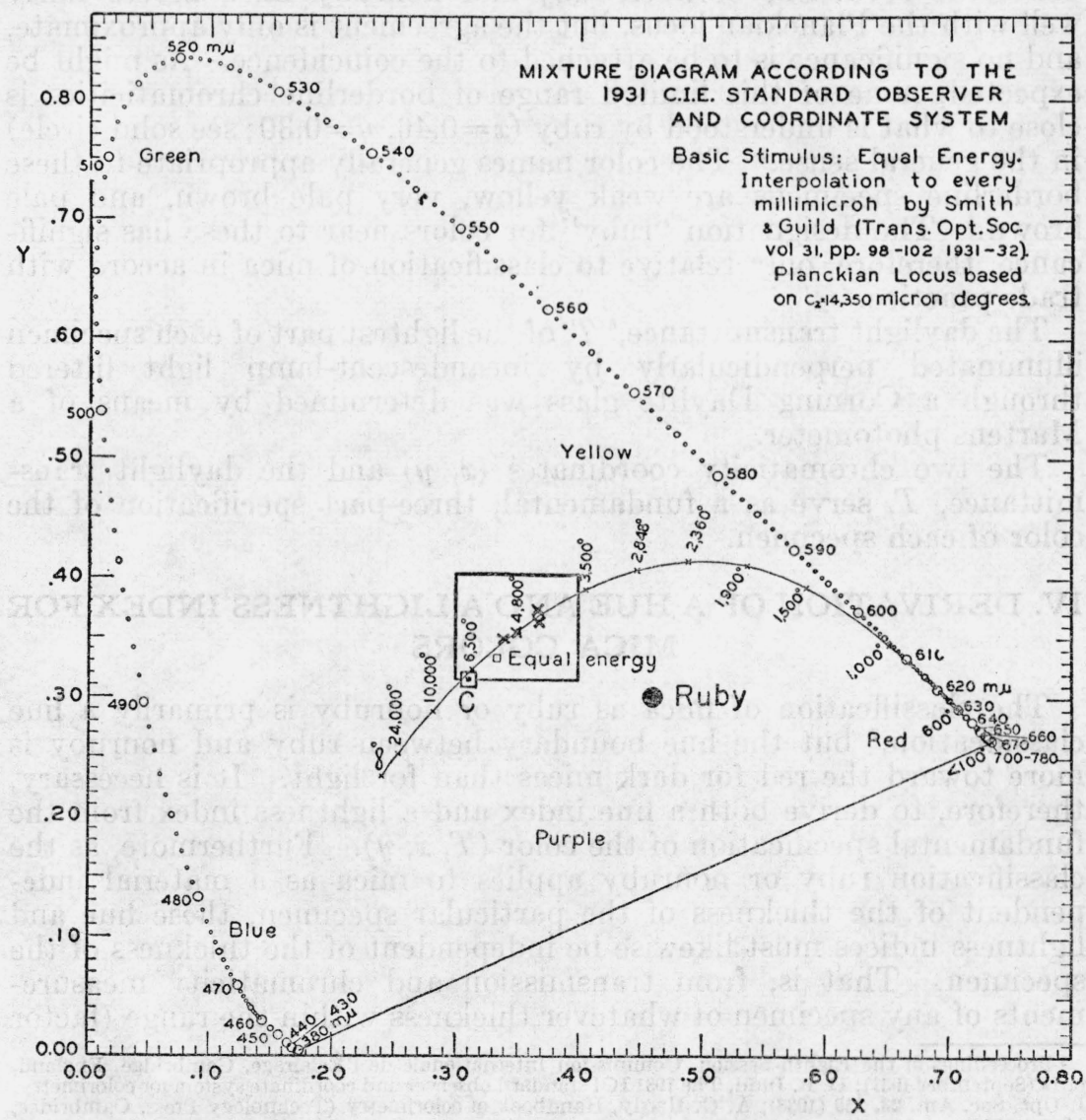

FIGURE 1-Chromaticity $(x, y)$ diagram showing borderline mica specimens (crosses) compared to standard illuminant $C$ (square), to ruby color (solid dot) and to the spectrum colors (small circles).

These mica specimens have chromaticities corresponding to weak yellow and pale brown, and are to be considered close to "ruby" only in accord with the meaning of the term in trade practice. These chromaticities by a coincidence happen to fall close to those of the ideal radiator at about $4,800^{\circ} \mathrm{K}$.

${ }^{2}$ D. B. Judd, Speciflcation of uniform color tolerances for textiles, Textile Research 9, 258 (1939). 


\section{Journal of Research of the National Bureau of Standards}

ard ICI colorimetric coordinate system. ${ }^{3}$ The chromaticity coordinates $(x, y)$ for each specimen were estimated for ICI standard illuminant $C$ (representative of average daylight) by adding the $x$-difference $(\Delta x)$ to the $x$-coordinate $(0.3101)$ for illuminant $C$, and by adding the $y$-difference $(\Delta y)$ to the $y$-coordinate $(0.3163)$; that is, $x$ for each specimen was computed as $0.3101+\Delta x$, and $y$ was computed as $0.3163+\Delta y$. Figure 1 shows the chromaticity diagram formed by plotting $y$ against $x$. The chromaticities of the standard specimens are shown by crosses in relation to those (small circles) of the spectrum and the point (indicated by a square) representing illuminant $C$, which corresponds to a colorless mica. It may be noted that the range of chromaticities covered by the five specimens is relatively small, and it happens that the points fall close to the Planckian locus in the neighborhood of $4,800^{\circ} \mathrm{K}$. Thus by a coincidence the borderline between ruby and nonruby mica agrees fairly well with the Planckian locus, but the agreement is only approximate, and no significance is to be attached to the coincidence. As might be expected, none of this limited range of borderline chromaticities is close to what is understood by ruby $(x=0.46, y=0.30$; see solid circle) in the general sense. ${ }^{4}$ The color names generally appropriate to these borderline specimens are weak yellow, very pale brown, and pale brown. ${ }^{5}$ The designation "ruby" for colors near to these has significance, therefore, only relative to classification of mica in accord with trade practice.

The daylight transmittance, ${ }^{\circ} T$, of the lightest part of each specimen illuminated perpendicularly by incandescent-lamp light filtered through a Corning Daylite glass was determined by means of a Martens photometer.

The two chromaticity coordinates $(x, y)$ and the daylight transmittance, $T$, serve as a fundamental, three-part specification of the color of each specimen.

\section{DERIVATION OF A HUE AND A LIGHTNESS INDEX FOR MICA COLORS}

The classification of mica as ruby or nonruby is primarily a hue classification, ${ }^{7}$ but the hue boundary between ruby and nonruby is more toward the red for dark micas than for light. It is necessary, therefore, to derive both a hue index and a lightness index from the fundamental specification of the color $(T, x, y)$. Furthermore, as the classification ruby or nonruby applies to mica as a material independent of the thickness of the particular specimen, these hue and lightness indices must likewise be independent of the thickness of the specimen. That is, from transmission and chromaticity measurements of any specimen of whatever thickness within the range (factor

\footnotetext{
3 Proceedings of the Eighth Session, Commission Internationale de l'Éclairage, Cambridge, England, p. 19 (September 1931); D. B. Judd, The 1931 ICI standard obser ver and coordinate system for colorimetry, J. Opt. Soc. Am. 23, 359 (1933); A. C. Hardy, Handbook of colorimetry (Technology Press, Cambridge, Mass., 1936).

Mee, for example, the color designated as ruby (6 G 6) by A. Maerz and M. Rea Paul, A dictionary of color, (McGraw-Hill Book Co., Inc., New York, N. Y., 1930).

$\checkmark$ D. B. Judd, and K. L. Kelly, Method of designating colors, J. Research NBS 23, 355 (1939) RP1239.

o Transmittance is the ratio of transmitted to incident flux; formerly, transmission. See Physical concepts; radiant energy and its measurement, Committee on Colorimetry, J. Opt. Soc. Am. 34, 188 (1944).

${ }_{7}$ Hue is the attribute of color that determines whether the color is red, yellow, green, blue, purple, or the like.

Lightness is the attribute that permits an object-color to be classified as equivalent to some member of the series of grays ranging between black and white. See ASTM Tentative Definitions of Terms Relating to Paint, Varnish, Lacquer and Related Products, D16-42T.
} 
of about 10) suitable for test, it should be possible to find the same hue and lightness indices within the uncertainties of the measurements.

As a basis for choosing functions of daylight transmittance, $T$, and chromaticity coordinates $(x, y)$ that could serve as these indices, measurements were made upon six specimens selected at random from five different mica mines ranging from a specimen found to be ruby by a large margin to one that is very green, and including some that are close to the border line. These specimens, except for $110 \mathrm{R}$ and $117 \mathrm{R}$ arbitrarily numbered, are identified by the number of the lot from which they were chosen followed by an " $R$ " to indicate that they are random choices. After each specimen was measured it was split so as to provide new specimens of about one-third and two-thirds, respectively, of the original thickness. And one such specimen after measurement was again split, yielding measurements at five different thicknesses. One specimen (No. 35) exhibited two distinct and uniform color areas side by side; these areas are designated " 35 red" and "35 green," respectively, and were measured separately.

TABLE 1.-Dependence of lightness index and hue index on thickness of specimen

\begin{tabular}{|c|c|c|c|c|c|c|c|}
\hline \multirow{2}{*}{$\begin{array}{l}\text { Specimen } \\
\text { No. }\end{array}$} & \multirow{2}{*}{$\begin{array}{l}\text { Thickness } \\
\text { (hun- } \\
\text { dredths of } \\
\text { an inch) }\end{array}$} & \multirow{2}{*}{$\begin{array}{l}\text { Daylight } \\
\text { transmit- } \\
\text { tance, } T\end{array}$} & \multirow{2}{*}{$\begin{array}{c}\text { Daylight } \\
\text { absorption } \\
\text { coefficient } \\
\text { (lightness } \\
\text { index) }\end{array}$} & \multicolumn{2}{|c|}{$\begin{array}{l}\text { Chromaticity coordi- } \\
\text { nates }\end{array}$} & \multirow{2}{*}{$\begin{array}{l}\text { Dominant } \\
\text { wavelength } \\
\quad(\text { in } \mu)\end{array}$} & \multirow{2}{*}{$\begin{array}{l}\text { Hue index, } \\
-\Delta G / \Delta Y\end{array}$} \\
\hline & & & & $x$ & $y$ & & \\
\hline \multirow{3}{*}{$6 \mathrm{R}}$. & $1.8_{4}$ & & & \multirow{14}{*}{$\begin{array}{r}0.385_{0} \\
.3578 \\
.339_{5} \\
.3277 \\
.322_{2} \\
.352_{5} \\
.337_{0} \\
.325_{8} \\
.375_{0} \\
.352_{8} \\
.333_{0} \\
.360_{1} \\
.342_{7} \\
.327_{5} \\
.408_{0} \\
.389_{b} \\
.337_{6} \\
.3917 \\
.371_{0}\end{array}$} & \multirow{14}{*}{ 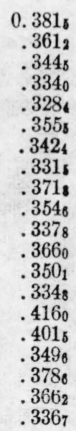 } & 579. 7 & \multirow{14}{*}{$\begin{array}{l}0.309 \\
.294 \\
.300 \\
.292 \\
.301 \\
.318 \\
.332 \\
.321 \\
.332 \\
.341 \\
.338 \\
.256 \\
.253 \\
.271 \\
.156 \\
.151 \\
.151 \\
.377 \\
.377 \\
.365\end{array}$} \\
\hline & $\begin{array}{l}1.16 \\
0.68\end{array}$ & $0.44_{1}$ & $0.27_{5}$ & & & $\begin{array}{l}578.5 \\
578.2\end{array}$ & \\
\hline & $\begin{array}{l}.42 \\
.26\end{array}$ & $\begin{array}{l}.678 \\
.760\end{array}$ & $\begin{array}{l}.29_{3} \\
.28\end{array}$ & & & $\begin{array}{l}577.5 \\
577.6\end{array}$ & \\
\hline $17 \mathrm{R}$ & $\begin{array}{l}1.20 \\
0.86\end{array}$ & 53 & 26. & & & 578.7 & \\
\hline & .40 & $.68_{3}$ & .260 & & & 578.0 & \\
\hline $35, \mathrm{re}$ & $\begin{array}{l}1.16 \\
1.18\end{array}$ & .597 & 15 & & & $\begin{array}{l}579.8 \\
579.1\end{array}$ & \\
\hline & 0.58 & .720 & .157 & & & 578.5 & \\
\hline 35 , green & 1. 18 & .564 & .17 & & & $\begin{array}{l}577.7 \\
577.1\end{array}$ & \\
\hline & 0.58 & $.70_{4}$ & .184 & & & 576.7 & \\
\hline $110 \mathrm{R}$. & $\begin{array}{l}2.41 \\
1.70\end{array}$ & $\begin{array}{r}0 y_{1} \\
.17_{4}\end{array}$ & $\begin{array}{l}.413 \\
.390\end{array}$ & & & $\begin{array}{l}577.5 \\
576.6\end{array}$ & \\
\hline & $0.6_{1}$ & $.51_{5}$ & .398 & & & 575.0 & \\
\hline & & & .140 & & & 581.4 & \\
\hline $117 \mathrm{R}$. & 2. 40 & $.42_{3}$ & .137 & & & 580.4 & \\
\hline & 0. & $.71_{5}$ & .1 & & & 578.9 & \\
\hline
\end{tabular}

Table 1 gives for these specimens not only the thickness in hundredths of an inch, the daylight transmittance, $T$, and the chromaticity coordinates $(x, y)$, but also the dominant wavelength computed from the chromaticity coordinates, ${ }^{8}$ and the ratio of the negative logarithm of internal transmittance to the thickness, $d$. The internal transmittance is obtained from the measured transmittance by correcting for the losses of light by reflection at the two surfaces and has been computed as $T / 0.90$, the constant 0.90 corresponding to the reflection losses for a plate of refractive index equal to 1.59 . The ratio of the negative logarithm of internal transmittance to the thickness may be called the daylight absorption coefficient.

Dominant wavelength is an index of hue that is expected to increase somewhat with increasing thickness of mica specimen, and daylight absorption coefficient is an index of lightness that is expected to

${ }^{8}$ OSA Committee on Colorimetry, The psychophysics of color, J. Opt. Soc. Am. 34, 265 (1944); see alsq footnote 3 . 


\section{Journal of Research of the National Bureau of Standards}

vary slightly with thickness of specimen. However, reference to table 1 shows that this latter variation is in fact less than the uncertainty of the determination; hence daylight absorption coefficient is a satisfactory index of the lightness or darkness of mica color. On the other hand, the expected increase in dominant wavelength is seen to be significant. For each of the six specimens, a regular increase may be noted greater than the uncertainty of the determination. It is worthwhile, therefore, to search for another hue index less dependent upon thickness of specimen.

On the chromaticity diagram of figure 1 constant dominant wavelengths correspond to straight lines intersecting at the point representing a colorless specimen $(x=0.3101, y=0.3163)$. The progressive increase of dominant wavelength with thickness is represented on a chromaticity diagram as a curved line similar to the Planckian locus. The points corresponding to the chromaticity coordinates $(x, y)$ are plotted in figure 2, which is the section of the $(x, y)$-diagram inclosed by the rectangle on figure 1 shown to an enlarged scale. The points corresponding to each specimen are connected by a smooth dotted line. The generally regular curvature toward red for increasing thickness is evident, and it appears from these specimens that the

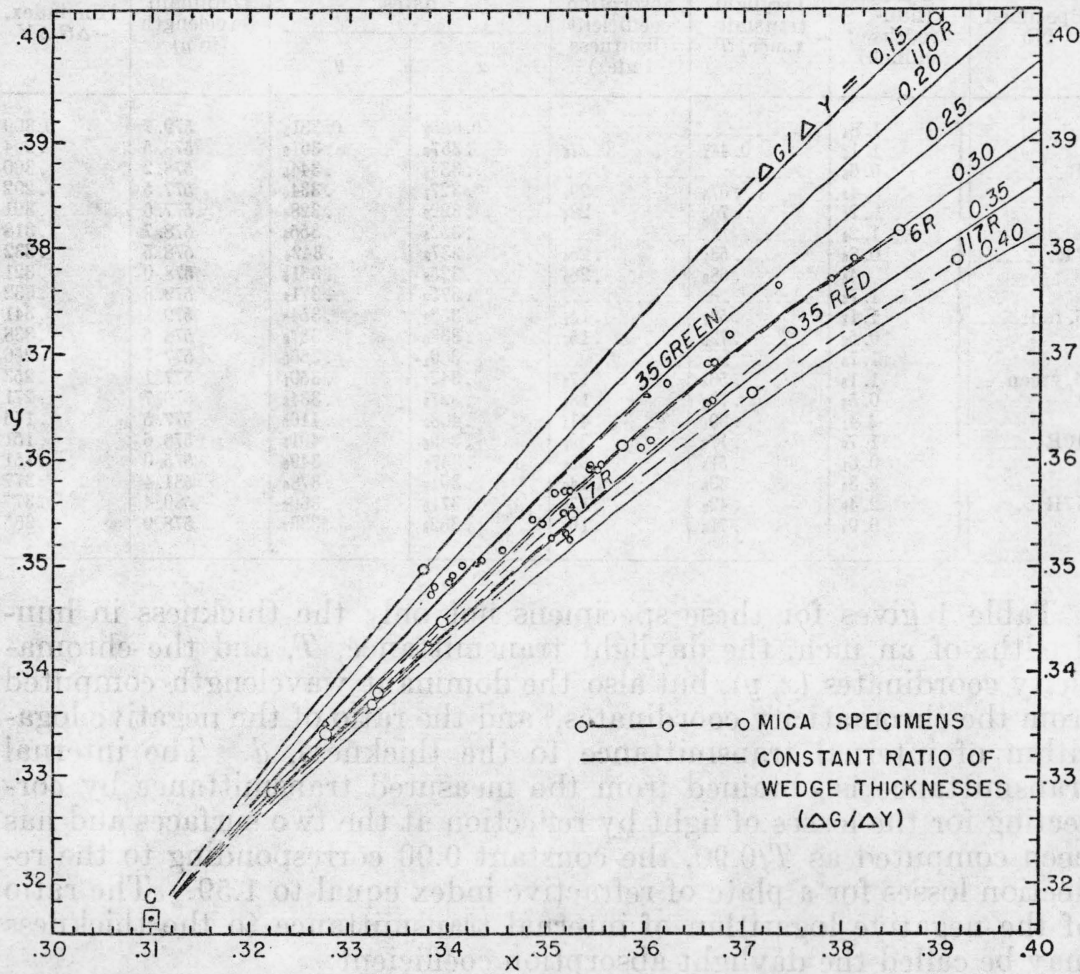

FIGURE 2.-Variation of the chromaticity of mica specimens (dashed lines) with thickness shown on an enlarged scale on a portion of the $(x, y)$ chromaticity diagram; see rectangle on figure 1 .

Closely the same chromaticity variations are produced on the colorimeter by holding constant the thickness -ratio of bluish green wedge to yellow wedge (solid lines), and on this account this ratio is used as the hue index for mica. 
direction of this curvature is constant and its amount reasonably constant. This direction corresponds to that characteristic of any light-absorbing medium whose spectral transmittance increases in a general way with wavelength. Each solid curve shown on figure 2 corresponds to a series of two-component filters, a yellow component and a red component, both of known spectral transmittance ${ }^{9}$, in which the ratio of thicknesses of the components is held constant, whereas the total thickness varies. The yellow component is that comprising the yellow wedge of the chromaticity-difference colorimeter. The red component is a hypothetical filter having the same effect in combination with the yellow component as that of the bluish-green wedge of the colorimeter combined with the specimen. The amount of the yellow component is indicated by the symbol $\Delta Y$, which is proportional to the thickness of yellow glass required for setting up a chromaticity match involving the mica specimen. The amount of the red component is similarly indicated by the symbol $-\Delta G$, which is proportional to the thickness of the bluish-green glass required to be combined with the mica specimen to duplicate the chromaticity of the yellow component. The minus sign is prefixed to the symbol $\Delta G$ to indicate that the bluish-green glass to produce a chromaticity match has to be combined with the mica specimen instead of with the yellow component. An unusually large value of $-\Delta G$ indicates an unusually reddish mica specimen.

Each solid curve of figure 2 is identified by the ratio of the amounts of the two components, $-\Delta G / \Delta Y$, expressed in terms of the arbitrary scales on the wedges. This ratio is taken as the hue index; and values of this hue index are given for all specimens in table 1 . It will be noted that the variations in hue index are irregular and therefore within the experimental uncertainty.

A lightness index and a hue index have therefore been found for mica, both indices being independent of thickness of the specimen. The lightness index is the ratio of the negative logarithm of internal transmittance of the mica specimen to its thickness, $[-\log (T / 0.90)] / d$. The hue index is the ratio of the red component to the yellow component in the filter required to duplicate the chromaticity of the mica specimen, $-\Delta G / \Delta Y$.

\section{DERIVATION AND VALIDIFICATION OF A COLOR STANDARD FOR RUBY MICA}

As a guide to the use of the hue and lightness indices in setting up a color standard for ruby mica, these indices have been determined not only for the 5 borderline specimens but also for representative specimens selected from the most doubtful of the 35 lots whose classification was reviewed by the industry task committee and for 4 specimens of ruby mica selected at the same time as the borderline specimens. Table 2 gives the results of these measurements in the same form as table 1 . Figure 3 shows on the $(x, y)$-diagram the chromaticities of these specimens together with those for two thicknesses of the "35 red" specimen see table 1. A straight line has been drawn between points representing the extreme borderline standards; this straight line is drawn solid between the extremes and is shown dashed beyond them as an approximate indication of the border line

\footnotetext{
D. B. Judd, Specification of uniform color tolerances for textiles, Textile Research 9, 260 (1939).
} 
between the chromaticities of ruby and nonruby mica. It will be noted that this straight line divides the mica specimens somewhat in accord with accepted color grading. Nonruby mica specimens tend to be represented above this line; ruby, below. There are exceptions, however; one measurement of No. 1-quality ruby standard yields a point on the wrong side of the line, two representative specimens from the nonruby lot No. 35 fall on the ruby side, the thick " 35 red" specimen falls on the green side. These exceptions, particularly the last one, are important, and indicate that the color grading of mica is not based upon this line. Furthermore, there is no simple curved line (such as the Planckian locus, see figure 1) to serve as a border line that will eliminate these discrepancies. This is the evidence that showed that the color grading of mica is based on other factors than chromaticity and led to the search for a lightness index.

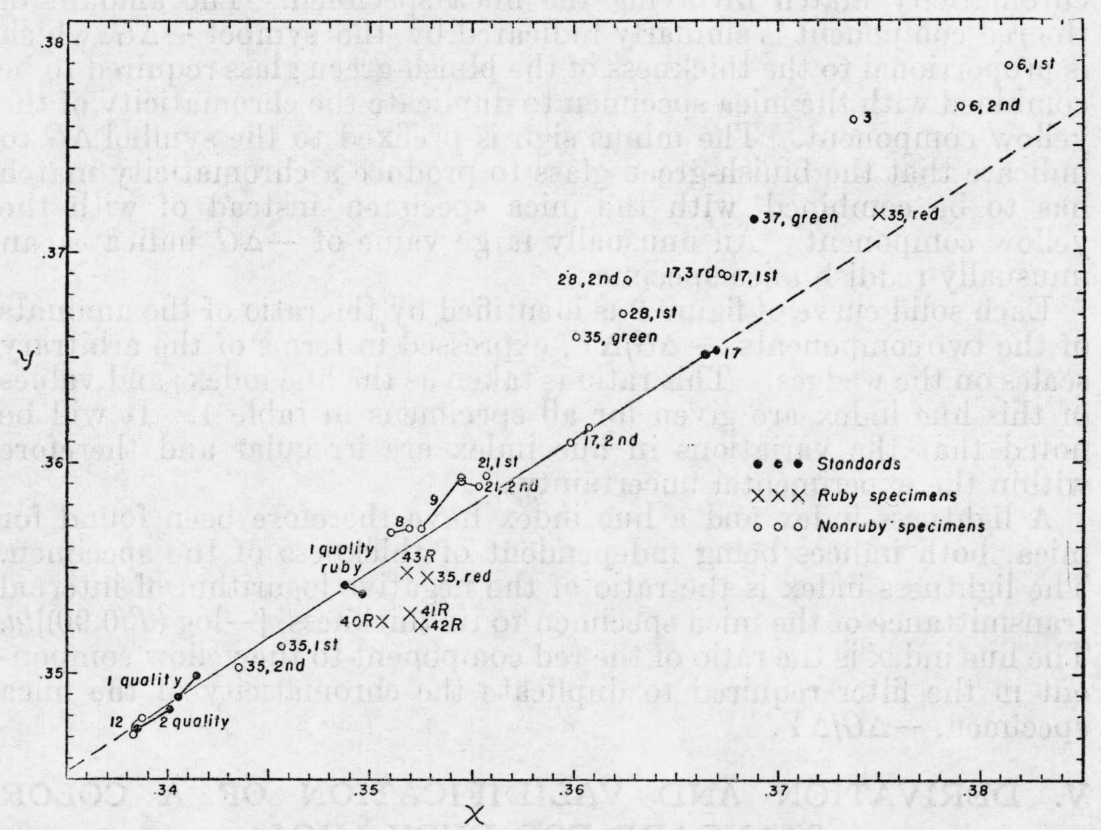

Figuke 3.-Correlation between chromaticity of mica specimens and accepted color classification of the lots from which the specimens were drawn.

The straight line separates the $(x, y)$ chromaticity diagram into an upper left and a lower right section which correlates roughly with the accepted color classification. An important exception is lot No. 35 composed partly of ruby and partly of nonruby mica; the " 35 red" specimen has a chromaticity represented on the same side of the line as the " 35 green" and other nonruby specimens (circles). This graph proves that color grading of mica according to trade practice is based partly but not wholly on chromaticity.

Figure 4 is a plot of lightness index against hue index for the same specimens shown in figure 3 . On this plot the lightness index is plotted with a descending scale, so that specimens of light-colored mica are represented near the top of the plot. Reddish mica is represented near the right of the plot; greenish, near the left. As on figure 3 a straight line has been drawn to connect the points representing the extremes of the borderline specimens. This line is shown solid as a basis for interpolation between the extremes, and is continued by dotted lines to indicate the most reasonable basis for extrapolation 
TABLE 2-Thickness, daylight transmittance, chromaticity coordinates, lightness index, and hue index for 5 standards and 23 mica specimens

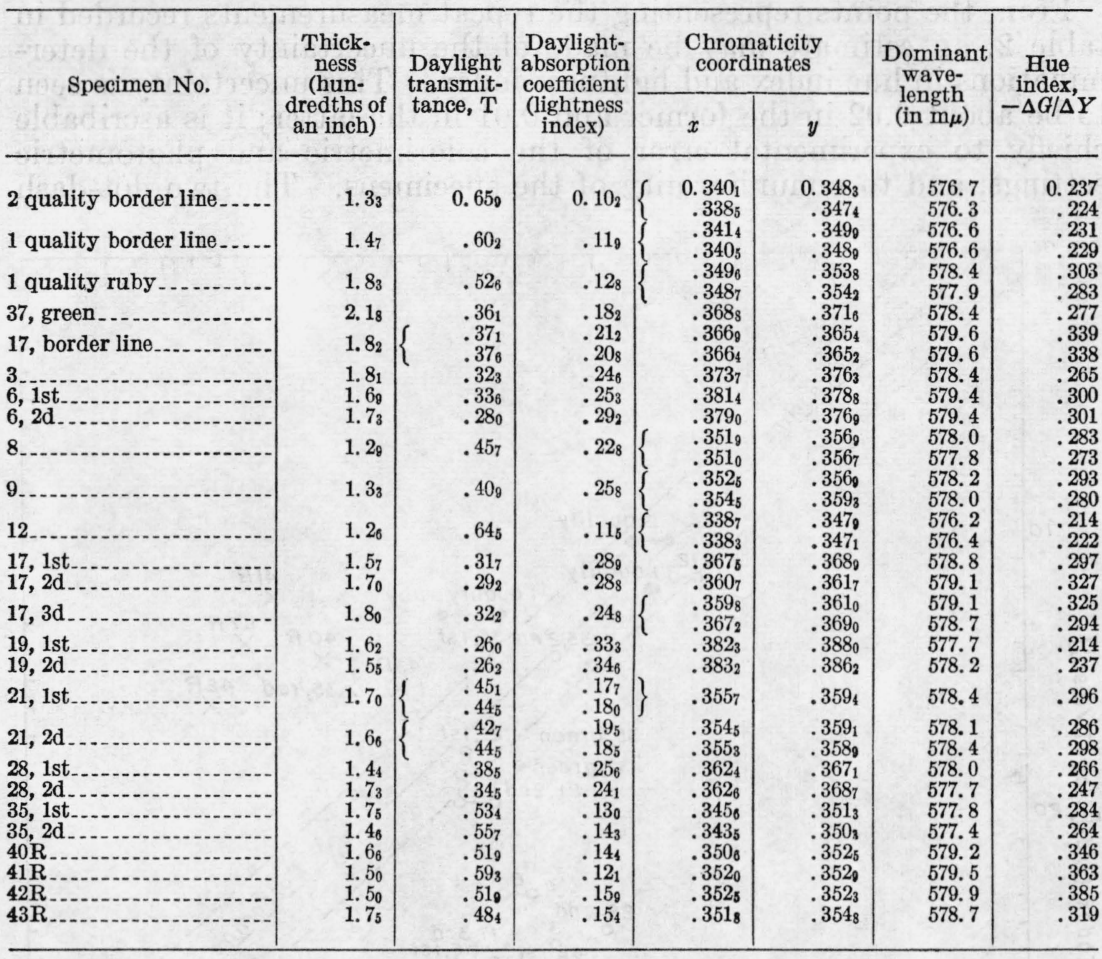

beyond the extremes represented by the borderline samples. It should be noted that this straight line departs considerably from the vertical; that is, the grading of mica as ruby or nonruby according to trade practice departs considerably from grading purely on the basis of hue alone. A specimen of light-colored mica can be considerably greener than the dark borderline specimen and still be graded ruby.

It should also be noted that the correlation between location of points on this diagram relative to the borderline and color grading by the industry task committee and experts of the Colonial Mica Corporation is nearly perfect. The "37 green" standard (solid circle) falls well below the border line. The "1 quality ruby" falls well above the border line. The " 35 red" specimen and the six specimens (40R to 43R) picked at random from lots graded "ruby" are all represented well above the border line. Seventeen of the 18 specimens chosen as representative of the lot graded by the industry task committee as nonruby are represented below the line, as are also the " 35 green" and " $110 \mathrm{R}$ " specimens. The only exception is the first specimen chosen as representative of lot No. 35; this specimen falls above the border line. It is worth noting that the second specimen chosen as representative of this lot is represented slightly below the line in accord with the judgment of the industry task committee. From this correlation it may be concluded that figure 4 is successful in reproducing 


\section{Journal of Research of the National Bureau of Standards}

the grading of mica into ruby and nonruby groups in accord with trade practice.

From the points representing the repeat measurements recorded in table 2 , an estimate may be made of the uncertainty of the determinations in hue index and lightness index. This uncertainty is seen to be about 0.02 in the former and 0.01 in the latter; it is ascribable chiefly to experimental error of the colorimetric and photometric settings, and to nonuniformity of the specimens. The two dot-dash

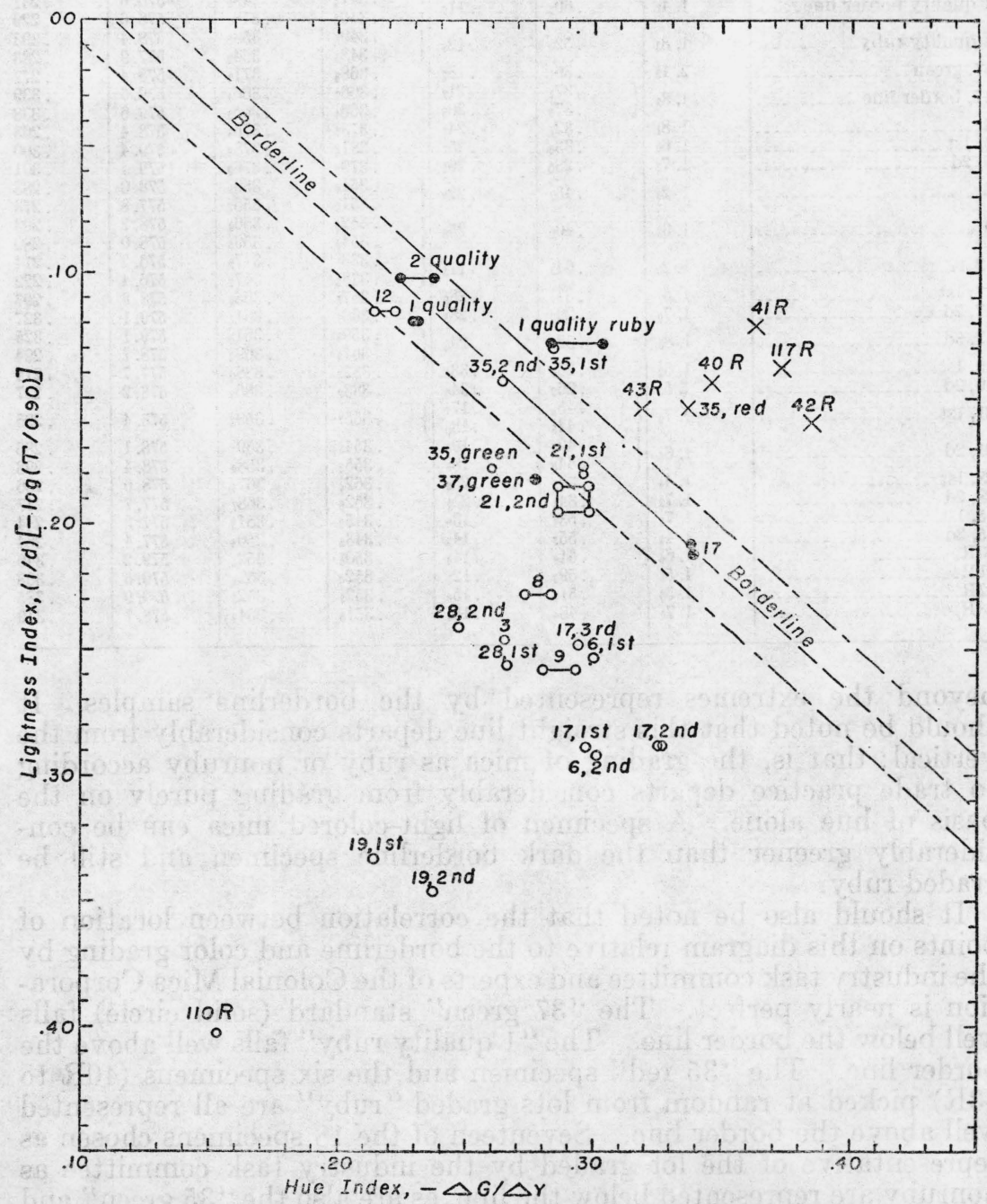

FIGURE 4.-Lightness indices of 3 borderline, 7 ruby and 19 nonruby mica specimens plotted against their hue indices.

The solid straight line passes close to the points representing the three borderline specimens. Note that the six specimens of ruby mica chosen at random (crosses) are represented above the line together with the " 1 quality ruby" standard. Also note that the " 37 green" standard and all but one of the specimens chosen as representative from nonruby lots of mica (circles) are represented below the line. The dot-dash lines represent color distinctions from the border line (solid and dotted) just distinguishable with certainty by one determination according to the method used. These lines are used as the basis for classifying lots of mica as ruby, nonruby, or border line. 
lines drawn on figure 4 therefore delineate what may be called a borderline area of the diagram. Mica specimens represented by points within this area are considered to be border line in the sense that it is not definitely known whether they should be classed as ruby or nonruby. It may be noted that all of the borderline specimens are represented by points within this area, as is also the first specimen selected as representative of lot No. 21 and the second specimen selected as representative of lot No. 35. Repeated measurements of these specimens would decide whether they are ruby or nonruby according to the arbitrary solid line shown; and such ropeat measurements were made on specimens drawn from lots No. 12 and No. 21. But so great is the variation in color within a given lot that it is usually more significant to draw another sample, choose a representative specimen, and measure that. Note that the third specimen from lot No. 17 differed in lightness index from the first and second specimens by nearly 0.04 , as did also the first and second specimens chosen from lot No. 6. Figure 4 has been used in the classification of mica samples into the three classes: ruby, nonruby, border line.

\section{PROCEDURE USED IN COLOR GRADING MICA}

1. From the lot to be graded there is selected, a few pieces at a time from 8 to 10 parts of the pile, a sample of approximately 1 pound.

2. The specimens comprising this sample are spread out, a few at a time, on a large white surface and arranged approximately according to luminous transmission; that is, all light-colored specimens are placed at one end of a series, dark-colored at the other end, and the intermediate specimens are also arranged approximately according to lightness of the color. Unusually reddish or unusually greenish specimens are set aside.

3 . By means of a direct-reading micrometer 20 specimens are selected with thicknesses between 0.015 and 0.020 inch. The arrangement in a light-to dark series facilitates this selection, and the casting out of unusually reddish and unusually greenish specimens insures that the specimens selected are representative of the sample. The required range of thicknesses is favorable for the measurements which differentiate the color of one lot of mica from another.

4. The 20 specimens so selected are spread out on a large white surface, illuminated by light from the north sky, and arranged by visual inspection into a two-dimensional array, light-colored specimens to the top, dark-colored to the bottom, reddish specimens to the right, greenish to the left.

5 . This array of colors is studied visually for nonuniformities in spacing, which might cause a centrally located specimen to be nonrepresentative in color, and a representative specimen is chosen with due regard to all 20 specimens.

6 . The thickness, $d$, and the daylight transmittance, $T$, are measured for the representative specimen as described in section III; the lightness index, $[-\log (T / 0.90)] / d$, is computed as described in section IV.

7. The chromaticity coordinates $(x, y)$ of the representative specimen for ICI illuminant $C$ are determined by whatever means are a vailable, the point corresponding to these coordinates is plotted on figure 3 , and the hue index, $-\Delta G / \Delta Y$, is read from the family of solid curves thereon; or, alternatively, if the chromaticity-difference colori- 
meter referred to in section III is used, the hue index, $-\Delta G / \Delta Y$, is found directly without obtaining the chromaticity coordinates.

8. The point defined by the lightness index plotted against the hue index on figure 4 determines the classification of the mica specimen measured. If the point falls between the two dot-dash lines, the specimen is classed as border line; if it falls above both, ruby; if below both, nonruby.

Analytically expressed, if $H$ be the hue index and $L$, the lightness index, the specimen is classed as ruby if $0.927 H-L-0.104$ is greater than 0.019 ; nonruby if $0.927 H-L-0.104$ is less than -0.019 ; and border line if $0.927 H-L-0.104$ is between 0.019 and -0.019 . The equation of the solid line on figure 4 is $0.927 H-L-0.104=0$.

9. The class of the lot of mica to be color graded is taken to be the same as the class of the specimen chosen as representative by steps 1 to 5 .

Washington, July 6, 1945. 